\title{
Analysis on Dissociation of Pyramidal I Dislocation in Magnesium by Generalized-Stacking-Fault Energy
}

\author{
Qun $\mathrm{Zu}^{1} \cdot$ Ya-Fang Guo ${ }^{1} \cdot$ Xiao-Zhi Tang ${ }^{1}$
}

Received: 3 December 2014/Revised: 19 January 2015/Published online: 5 May 2015

(C) The Chinese Society for Metals and Springer-Verlag Berlin Heidelberg 2015

\begin{abstract}
The generalized-stacking-fault energies are calculated to illustrate the dissociation of $\langle c+a\rangle$ dislocation on pyramidal I plane in magnesium. The $\gamma$ surfaces of $\{10 \overline{1} 1\}$ plane and its adjacent planes $\{30 \overline{3} 4\}$ and $\{30 \overline{3} 2\}$ are presented using Liu embedded-atom-method potential method, and one possible dissociation path of $1 / 3\langle 11 \overline{2} 3\rangle$ dislocation on $\{10 \overline{1} 1\}$ plane with minimum energy is predicted. Meanwhile, another two reasonable dissociation paths of $1 / 3\langle 11 \overline{2} 3\rangle$ dislocation successively on $\{30 \overline{3} 4\}$ and $\{30 \overline{3} 2\}$ planes are also proposed. Moreover, based on molecular dynamics simulations of magnesium single crystals under $c$-axis compression, the possible slip path is further examined and discussed.
\end{abstract}

KEY WORDS: Generalized-stacking-fault energies (GSFEs); Molecular dynamics (MD); $\langle c+a\rangle$ slip; Dissociation; Magnesium

\section{Introduction}

It is well known that magnesium and its alloys are the lightest among the light metal materials for structural application. However, due to its hexagonal close-packed (hcp) crystallographic structure, the formability of magnesium is restricted. The limited number of available slip systems makes its plastic deformation more difficult. In the research of hcp structures, it is widely recognized that the slip and twinning are the dominant deformation modes, especially, more attentions are focused on the $\langle c+a\rangle$ slip in pyramidal I and II planes [1-9]. There are two focuses on the discussion of these two pyramidal $\langle c+a\rangle$ slips: (1) Which slip is preferred? The pyramidal II slip system for

Available online at http://link.springer.com/journal/40195

Ya-Fang Guo

yfguo@bjtu.edu.cn

1 Institute of Engineering Mechanics, Beijing Jiaotong University, Beijing 100044, China zinc [10] and cadmium [11] has been reported under hexagonal axis compression, but no such slip could be evidenced for magnesium in Yoshinaga's experiment [1]. In Guo's compressive simulations of bulk single crystals [12], the initial slip could be identified as the $\{11 \overline{2} 2\}$ slip for titanium, but the $\{10 \overline{1} 1\}$ slip for magnesium. From $c$-axis micro-compression experiments in magnesium, Byer et al. [2] claimed pyramidal II dislocations remained questionable and confirmed the presence of pyramidal I dislocations. However, Galiyev et al. [4] have found pyramidal II slip traces using scanning electron microscopy (SEM) in magnesium alloy ZK60. Lilleodden et al. [5] have suggested that six active pyramidal II slip systems played an important effect on plasticity and hardening in magnesium micro-pillar compression by electron backscattered diffraction (EBSD) measurements. (2) How does the pyramidal $\langle c+a\rangle$ dislocation dissociate? Dissociations of both pyramidal I and II dislocation cores have been widely investigated in 1980s and 1990s [13-18]. In recent years, $\mathrm{Li}$ and $\mathrm{Ma}$ [19] revealed a slip mode consisting of other two separate partial dislocations $(1 / 4\langle 01 \overline{1} 2\rangle$ and 
$1 / 4\langle 01 \overline{1} 2\rangle+1 / 6\langle 2 \overline{1} \overline{1} 0\rangle)$ in pyramidal I plane that nucleates independently in magnesium single crystal by MD simulations. Ando et al. [7] have exhibited that the pyramidal II dislocation had two stable core structures at $0 \mathrm{~K}$. Furthermore, transmission electron microscopy (TEM) results showed that the pyramidal II dislocations were presented as either straight lines or with a zigzag configuration after $c$-axis compression [9].

Up to now, it is still unclear on the pyramidal $\langle c+a\rangle$ slip mechanisms. Noteworthily, the above-mentioned discussions are largely focused on the discrepancy between simulative results revealing predominantly pyramidal I slip and micro-experiments showing mainly pyramidal II slip. In Guo's MD simulations [20], the pyramidal I dislocations combined by two successive partial dislocations of $1 / 6[20 \overline{2} \overline{3}]$ and $1 / 6[02 \overline{2} \overline{3}]$ were responsible for the plastic deformation in magnesium under the $c$-axis compression. This simulation result was not consistent with Lilleodden et al.'s experimental observation [5] that pyramidal II slips dominated the plasticity in magnesium single crystal. More recently, Tang et al. [21] have revealed that pyramidal II $\langle c+a\rangle$ dislocations were obtained in molecular simulations with a 3D large-scale model, but pyramidal II slip usually occurred later after the pyramidal I slip. Thus, it is suggested that the pyramidal I dislocation occurs more easily in the initial plasticity deformation in magnesium.

Analysis of the generalized-stacking-fault energy (GSFE) is useful for understanding the slip mode and twinning mechanism [22]. The stacking fault energy (SFE) is the intrinsic parameter of materials. Both the stable and unstable SFEs are important factors on the twinnability and the nucleation, mobility and stability of dislocations [23]. The stability of translational stacking faults on basal and non-basal planes of magnesium single crystal has been investigated by atomic potentials simulation [14, 24-26], ab initio [24-27] and experiment methods [28, 29]. Summaries of prism unstable SFEs along $\langle 11 \overline{2} 0\rangle$ direction were shown in Yasi's work [24]; it is shown that the results of SFEs in experiments were discrepant with those obtained by atomic potentials simulations and ab initio calculations. Recently, two successive papers presented the core structures of screw and edge dislocations on the basal, prism [24] and pyramidal planes [25] in magnesium by the embedded-atom-method (EAM) interatomic potentials and $a b$ initio method. The stable stacking fault structures and the possible slip paths were given.

Due to the hcp structure, the pyramidal I plane in magnesium is a super-plane consisted of two closed-packed planes $\left(A^{\prime}\right.$ and $\left.B^{\prime}\right)$ as shown in Fig. 1. Therefore, the possible slip paths for the pyramidal I slip are proposed on either the $\{10 \overline{1} 1\}$ plane $[14,19]$ or its adjacent $\{30 \overline{3} 4\}$ and $\{30 \overline{3} 2\}$ planes $[6,30,31]$. In this paper, the GSFEs of both (1011) plane and its adjacent $(30 \overline{3} 4)$ and $(30 \overline{3} 2)$ planes in magnesium are calculated using EAM potential method. We focus on the study of the dissociation of pyramidal I $\langle c+a\rangle$ dislocation in magnesium single crystals. The possible dissociation mechanisms of pyramidal I dislocation are discussed based on theoretical analysis and tested by MD simulations.

\section{Simulation Model and Method}

\subsection{Atomic Configuration of Pyramidal I Planes}

In Fig. 1, the lattice structure of magnesium single crystal and the orientations are shown. It can be seen that the atoms align in layers along the $[\overline{1} 2 \overline{1} 0]$ direction periodically

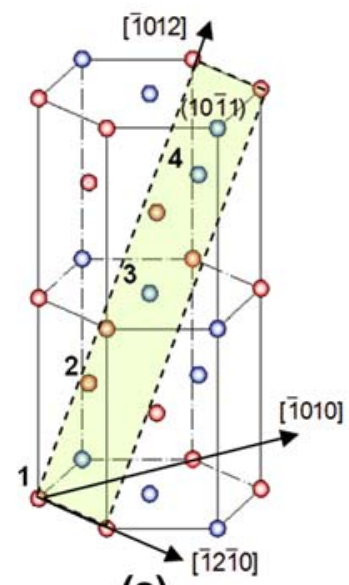

(a)

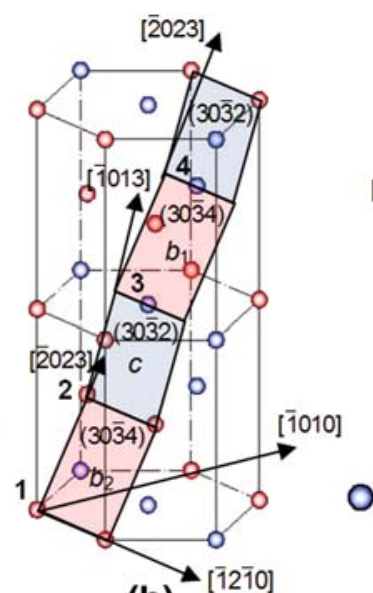

(b)

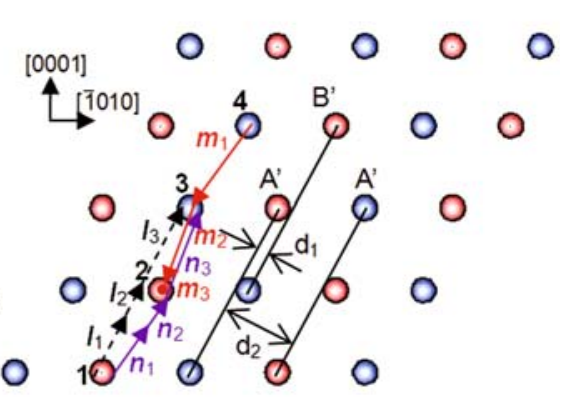

(c)

Fig. 1 Lattice structure of magnesium single crystal: a a pyramidal $\mathrm{I}(\pi 1)$ plane $(x=[\overline{1} 2 \overline{1} 0], z=[\overline{1} 012])$ is marked by light green; b two

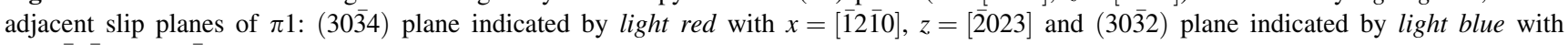
$x=[\overline{1} 2 \overline{1} 0], z=[\overline{1013}] ; \mathbf{c}$ atoms configuration of $\pi 1$ super-planes and possible slip vectors 
with the sequence of ... $\mathrm{ABAB} \ldots$ : atoms in layer $\mathrm{A}$ are marked as red circles, and atoms in layer $B$ as blue circles. The (1011) pyramidal I plane and its two adjacent slip planes $(30 \overline{3} 4)$ and $(30 \overline{3} 2)$, which are marked by light green, red and blue, respectively, are displayed in Fig. 1a, b. Actually, the $\pi 1$ super-plane consists of two adjacent planes $\left(A^{\prime}\right.$ and $\left.B^{\prime}\right)$, as shown in Fig. 1c. The distance $d_{1}$ between the nearest neighboring $A^{\prime}$ and $B^{\prime}$ planes is far closer than that $\left(d_{2}\right)$ between two neighboring $A^{\prime}$ or $B^{\prime}$ planes. Thus, the slips of pyramidal I plane are expected to occur between two super-planes, as (3034) and (30 $\overline{3} 2)$ planes.

\subsection{Calculation of GSFE}

The usual approach to calculate the energy surface $(\gamma)$ is shown in Fig. 2. Our simulation model uses $y$ coordination normal to glide plane and $x o z$ plane parallel to the glide plane. Periodic boundary condition is applied in $x$ and $z$ directions, while free boundary condition is used in $y$ direction. The top half of the crystal is moved with respect to the bottom half by the slip vector $\boldsymbol{u}$, which can be defined as

$\boldsymbol{u}=p \boldsymbol{x}+q \boldsymbol{z}$,

with $0 \leq p \leq 1, \quad 0 \leq q \leq 1$. For each relative displacement, all atoms are relaxed to minimize the energy with $x$ and $z$ directions fixed, and $y$ direction relaxed. The stacking fault energy $E$ is defined as

$E=\frac{E_{\mathrm{sf}}-E_{\mathrm{un}-\mathrm{sf}}}{A}$,

noting that $E_{\mathrm{sf}}$ and $E_{\mathrm{un}-\mathrm{sf}}$ are the total energies of super cell with and without stacking fault, respectively, and $A$ is the area of stacking fault plane. As shown in Fig. 1, for (1011)

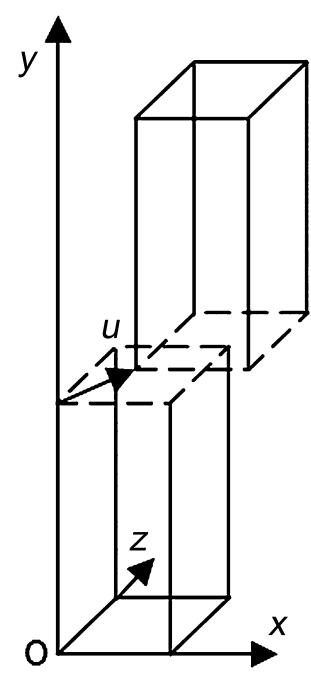

Fig. 2 The schematic of GSF structure plane calculation, the orientation has $x=[\overline{1} 2 \overline{1} 0], y=[10 \overline{1} 1]$, $z=[\overline{1} 012]$.While for its adjacent $(30 \overline{3} 4)$ and $(30 \overline{3} 2)$ planes, the orientation has $x=[\overline{1} 2 \overline{1} 0], y=[30 \overline{3} 4], z=[\overline{2} 023]$ and $x=[\overline{1} 2 \overline{1} 0], y=[30 \overline{3} 2], z=[\overline{1} 013]$ respectively.

The LAMMPS code [32] and the EAM potential developed by Liu et al. [33] are used. To validate the EAM potential, we calculated the GSFEs along $\langle 11 \overline{2} 3\rangle$ direction in pyramidal II plane using both Liu EAM potential and ab initio method with the Vienna ab initio simulation package (VASP) $[34,35]$. The stable SF structure and two unstable SF structures have energies of 240, 246 and $519 \mathrm{~mJ} / \mathrm{m}^{2}$ for the Liu EAM potential, and 197, 205 and $502 \mathrm{~mJ} / \mathrm{m}^{2}$ for ab initio method. Thus, the data by Liu EAM potential are fit well to those obtained by $a b$ initio method. Furthermore, the related results of GSFEs in basal, prismatic and pyramidal planes obtained by other computer simulations with different potentials [24-26] also confirmed the reliability of Liu EAM potential. In this work, the simulation is performed at $T=0 \mathrm{~K}$ for the pyramidal I plane. Dimensions of about $1.3 \mathrm{~nm} \times 29.1 \mathrm{~nm} \times 2.4 \mathrm{~nm}$ $(4672$ atoms $)$ and $1.3 \mathrm{~nm} \times 22.5 \mathrm{~nm} \times 1.9 \mathrm{~nm}(2400$ atoms) are used for calculating $\gamma$ surfaces of pyramidal I and its adjacent planes, respectively. The convergence of the energy is reached, and error in the total energy is less than $10^{-8} \mathrm{eV}$.

\section{Results and Discussion}

\subsection{GSFEs of $\{10 \overline{1} 1\}$ Plane}

The $\gamma$ surface of (1011) plane is shown in Fig. 3a. The straight slip path (path 1) and the minimum energy path (path 2) for $\{10 \overline{1} 1\}$ slip are indicated by black solid and dash lines, respectively. An unstable energy $\left(350 \mathrm{~mJ} / \mathrm{m}^{2}\right)$ exists on the path 1 when the shear displacement equals to $0.4 \mathrm{~nm}$ as shown in Fig. 4. Along the zigzag path 2, the maximum and minimum energies are about 300 and $120 \mathrm{~mJ} / \mathrm{m}^{2}$, which are both less than $350 \mathrm{~mJ} / \mathrm{m}^{2}$ on the straight path 1 . Thus, the $\langle c+a\rangle$ slip on pyramidal I plane may occur along path 2 by three steps of $\boldsymbol{l}_{1}(1 / 6[1012]), \boldsymbol{l}_{2}$ $(1 / 12[\overline{3} 4 \overline{1} 2])$ and $\boldsymbol{l}_{3}(1 / 4[\overline{1} 012])$ sequentially, as indicated by black arrows in Fig. 1c. Thus, the slip vectors can be described by

$$
1 / 6[\overline{1} 012]+1 / 12[\overline{3} 4 \overline{1} 2]+1 / 4[\overline{1} 012] \rightarrow 1 / 3[\overline{2} 113] .
$$

The $\gamma$ surface of $\{10 \overline{1} 1\}$ plane has also been calculated using Sun EAM potential and $a b$ initio methods by Nogaret et al. [25]. In their study, although the GSFEs with Sun potential are slightly higher than those using Liu potential and ab initio methods, the minimum energy path is almost consistent with our result. 

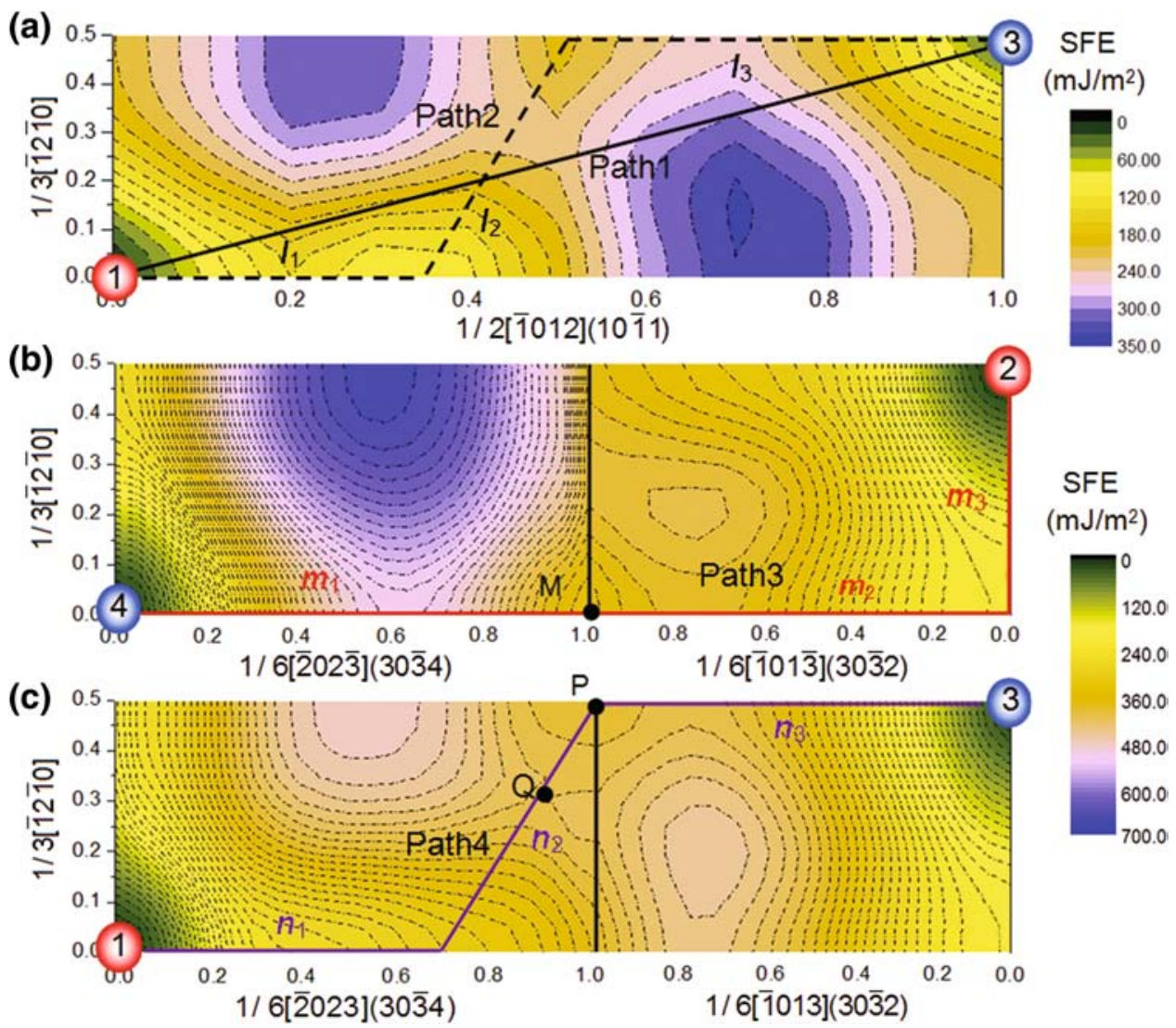

Fig. 3 The projection of $\gamma$ surfaces obtained using Liu EAM potential: a $\gamma$ surface of (1011) plane. The straight slip path and the minimum

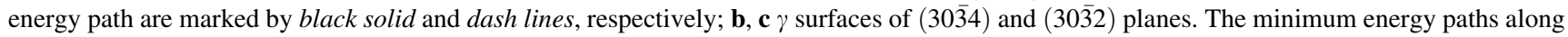
different slip planes and directions are marked by red and purple lines, respectively

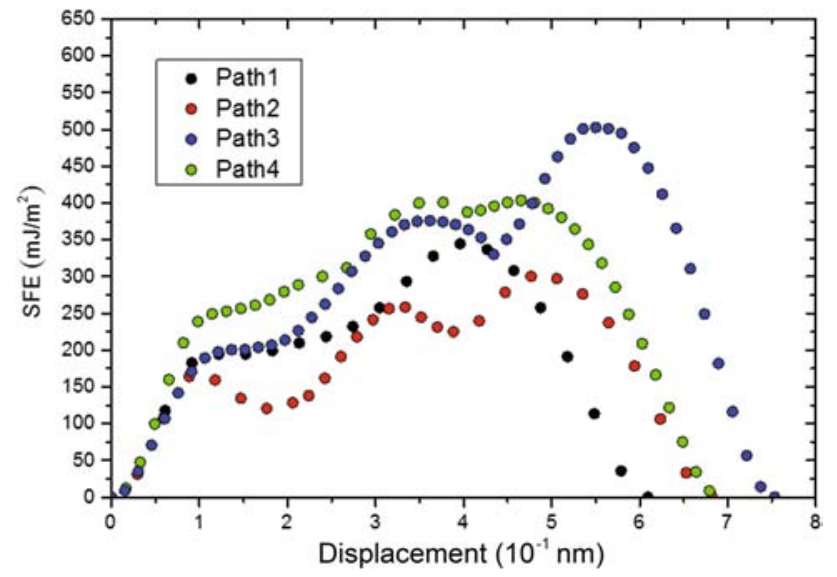

Fig. 4 The graph of GSFEs along possible paths of $\langle c+a\rangle$ slips

The $\gamma$ surfaces of $(30 \overline{3} 4)$ and $(30 \overline{3} 2)$ planes are sensitive to the initial slip plane and direction. Therefore, as indicated in Fig. 1c, there are two possible slip paths for the glide on $(30 \overline{3} 4)$ and $(30 \overline{3} 2)$ planes, as red arrows from atom 4 to 2 and purple arrows from atom 1 to 3 . If the initial slip plane is $b_{1}$ and the following slip plane is $c$ (from atom 4 to 2), the $\gamma$ surface is displayed in Fig. 3b. Obviously, a minimum energy point $(M)$ appears after the $1 / 6[\overline{2} 02 \overline{3}]$ slip is finished. Therefore, the minimum energy path to fulfill $\langle c+a\rangle$ slip, which is marked by red line (path 3), moves along three vectors of $\boldsymbol{m}_{1}, \boldsymbol{m}_{2}$ and $\boldsymbol{m}_{3}$, namely $\quad \boldsymbol{m}_{1}=1 / 6[\overline{2} 02 \overline{3}], \quad \boldsymbol{m}_{2}=1 / 6[\overline{1} 01 \overline{3}] \quad$ and $\boldsymbol{m}_{3}=1 / 6[\overline{1} 2 \overline{1} 0]$. The $1 / 3\langle\overline{2} 11 \overline{3}\rangle$ dislocation is expected to occur by dissociation reaction of

$1 / 6[\overline{2} 02 \overline{3}]+1 / 6[\overline{1} 01 \overline{3}]+1 / 6[\overline{1} 2 \overline{1} 0] \rightarrow 1 / 3[\overline{2} 11 \overline{3}]$.

In contrast, if the prior slip plane is $b_{2}$ and the following slip plane is $c$ (from atom 1 to 3 ), the $\gamma$ surface is exhibited in Fig. 3c. The slip path indicated by purple line (path 4) is the minimum energy way to fulfill $\langle c+a\rangle$ slip. No minimum appears in $1 / 6[\overline{2} 023]$ direction, whereas there is a minimum point $(\mathrm{P})$ and a saddle point $(Q)$ in the diagonal direction. After the completion of $m / 6[\overline{2} 023](m \approx 2 / 3)$ dislocation glide, the slip with the Burgers vector of $(1-$ $m) / 6[\overline{2} 023]+1 / 6[\overline{1} 2 \overline{1} 0]$ (along diagonal direction) follows. Then, a $1 / 6[\overline{1} 013]$ slip occurs to fulfill the $\langle c+a\rangle$ slip. Thus, the $1 / 3\langle\overline{2} 113\rangle$ slip can be approximately expressed by 


$$
\begin{aligned}
& m / 6[\overline{2} 023]+((1-m) / 6[\overline{2} 023]+1 / 6[\overline{1} 2 \overline{1} 0])+1 / 6[\overline{1} 013] \\
& \quad \rightarrow 1 / 3[\overline{2} 113],
\end{aligned}
$$

with $m \approx 2 / 3$. Three slip vectors, namely $\boldsymbol{n}_{1}=m / 6[\overline{2} 023]$, $\boldsymbol{n}_{2}=(1-m) / 6[\overline{2} 023]+1 / 6[\overline{1} 2 \overline{1} 0]$ and $\boldsymbol{n}_{3}=1 / 6[\overline{1} 013]$, are also marked in Fig. 1c. In addition, the GSFE curves of the possible slip paths are presented in Fig. 4. The maximum and minimum energies in path 3 are about 500 and $330 \mathrm{~mJ} /$ $\mathrm{m}^{2}$, respectively, and in path 4 are about 400 and $387 \mathrm{~mJ} /$ $\mathrm{m}^{2}$. Although the maximum energy in path 3 is slightly higher than that in path 4 , the more stable partial dislocation with lower energy also occurs in path 3. In the Sects. 3.2 and 3.3, discussions on which path is more reasonable will be presented.

\subsection{Discussions on Different Paths}

The above analysis reveals the possible pyramidal I $\langle c+a\rangle$ slip paths from the view of GSFEs. When the $\langle c+a\rangle$ slip occurs in an ideal crystal model, the path 2 is the preferred way due to the low energy. The conclusion is consistent with the simulations of Bacon and Liang [14] that they studied the pyramidal slip on the $\{10 \overline{1} 1\}$ plane of the hexagonal close-packed structure with pair-wise potentials. Two stable faults were found in hcp models, and the $1 / 3\langle\overline{1} 123\rangle$ dislocation was expected to extend over the $\{10 \overline{1} 1\}$ plane by dissociation reaction of

$$
1 / 4[\overline{1} 012]+1 / 12[1 \overline{4} 32]+1 / 6[\overline{1} 012] \rightarrow 1 / 3[\overline{1} \overline{1} 23] .
$$

Moreover, Bacon and Liang [14] also referred that the volume expansion had the effect on the $\langle c+a\rangle$ dislocation

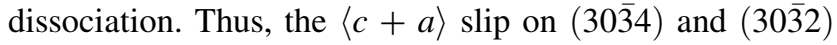
planes in our paper is approximated reasonably.

Other dissociations of $\langle c+a\rangle$ dislocation have also been presented in previous researches. For example, under tensile strain along the $c$-axis, $\mathrm{Li}$ and $\mathrm{Ma}$ [19] suggested a dissociation process of the pyramidal $\mathrm{I}\langle c+a\rangle$ dislocation on $\{0 \overline{1} 11\}$ plane in magnesium:

$2 \times\left(\frac{1}{2} \cdot \frac{1}{2}[01 \overline{1} 2]\right)+\frac{1}{2} \cdot \frac{1}{3}[2 \overline{1} \overline{1} 0] \rightarrow \frac{1}{3}[11 \overline{2} 3]$.

The same EAM potential was used in Kim's work [30], but the dissociation was quite different. In the nano-textured magnesium, the $\langle c+a\rangle$ slip occurred with the dissociation reaction of

$$
1 / 9[\overline{1} 013]+1 / 18[2 \overline{6} 43]+1 / 6[\overline{2} 023] \rightarrow 1 / 3[\overline{1} \overline{1} 23] .
$$

Furthermore, Jones and Hutchinson have given the same dissociation as Eq. (8) in Ti-6Al-4V alloy [31]. In the cold rolling experiment of $\mathrm{Mg}-\mathrm{Y}$ alloys, the associated $\langle c+a\rangle$ Burger vectors were observed by TEM [6] as

$$
1 / 3[11 \overline{2} 3] \rightarrow 1 / 6[20 \overline{2} 3]+1 / 6[02 \overline{2} 3] \text {. }
$$

In Eqs. (8) and (9), the slips on $\{30 \overline{3} 4\}$ and $\{30 \overline{3} 2\}$ planes are presented. Meanwhile, the $1 / 6\langle 20 \overline{2} 3\rangle$ partial dislocation appears in both Eqs. (8) and (9) due to the dissociation of the $1 / 3\langle\overline{1} \overline{1} 23\rangle$ dislocation. This is consistent with our calculation of $\gamma$ surface shown in Fig. $3 b$ that the $1 / 6\langle 20 \overline{2} 3\rangle$ dislocation is suggested to be relatively stable.

In conclusion, due to different simulation and experiment conditions, the dissociation of $\langle c+a\rangle$ dislocation varies. However, it can be concluded from above analysis that the pyramidal $\mathrm{I}\langle c+a\rangle$ slips occur either on the $\{10 \overline{1} 1\}$ plane or on its adjacent planes of $\{30 \overline{3} 4\}$ and $\{30 \overline{3} 2\}$.

\subsection{Atomistic Simulation of Pyramidal Slips Under $c$-Axis Compression}

For the hcp magnesium, the glide of $\langle a\rangle$ dislocations on the (0001) plane is easier to occur than the prismatic and pyramidal slips. In general, $\langle c+a\rangle$ slip may occur when the basal slip is restricted. Under $c$-axis tension simulations, both twinning and non-basal slips are found to play an important role on the deformation of magnesium single crystals [36, 37]. However, it was confirmed that there no twins were observed in magnesium single crystal under $c$ axis compression using molecular dynamics [20] and experiment methods [5]. Therefore, the atomic evolution simulation in this section is applied to investigate the atomistic details of the pyramidal $\langle c+a\rangle$ slip under $c$-axis compression.

To observe pyramidal I slip more clearly and reduce computational cost, a two-dimensional (2D) simulation model is used. As shown in Fig. 5, a uniaxial strain loading is exerted on the $c$-axis ([0001] direction) by applying a constant strain on the 1.0-nm-thick top layer and fixing the 1.0-nm-thick bottom layer. Periodic boundary and free boundary conditions are applied in $[\overline{1} 2 \overline{1} 0]$ and $[\overline{1010}]$ directions, respectively. The system with dimension of $1.3 \mathrm{~nm} \times 31.1 \mathrm{~nm} \times 16.6 \mathrm{~nm}$ containing about 30,000 atoms is studied. The simulation temperature is $5 \mathrm{~K}$. For each increasing strain, atoms are fully relaxed, and the evolution of atomic positions at different loading levels is investigated. To verify simulated results, similar simulations are carried out at 0 and $100 \mathrm{~K}$, and the sample with $2.6 \mathrm{~nm} \times 35.5 \mathrm{~nm} \times 17.7 \mathrm{~nm} \quad$ and $1.3 \mathrm{~nm} \times 25.9 \mathrm{~cm} \times 12.7 \mathrm{~nm}$ is tested. In all cases, the same dissociation of pyramidal I dislocation is obtained.

The forming process of the $1 / 3[\overline{2} 11 \overline{3}]$ dislocation under $c$-axis compression is shown in Fig. 5. Three different configurations of the pyramidal slip are indicated by common neighbor analysis (CNA) [38] and coordinates along [12 10 ] direction in Fig. 5a, b, severally. The slip vectors are indicated in Fig. 5c. In our $c$-axis loading 


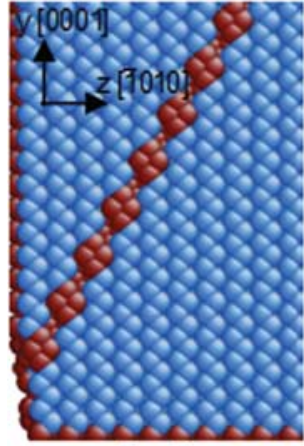

(a-1)

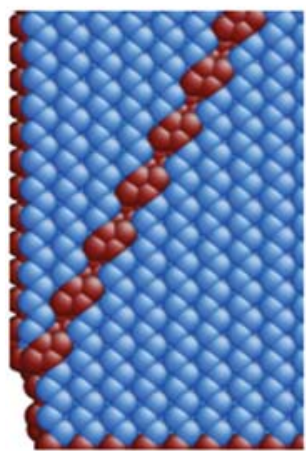

(a-2)

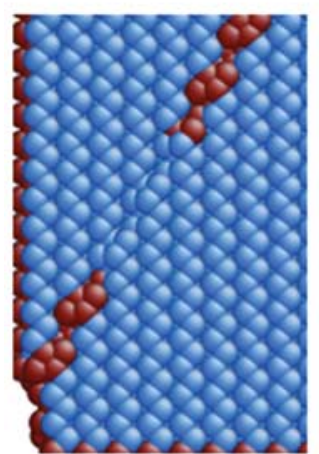

(a-3)

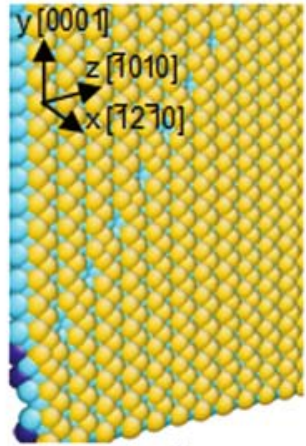

(b-1)

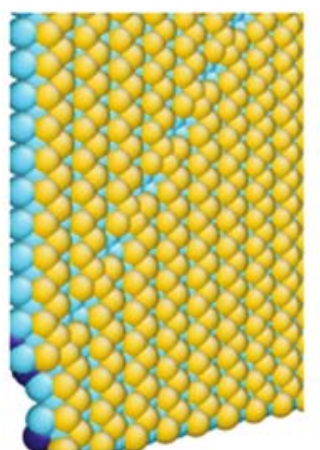

(b-2)

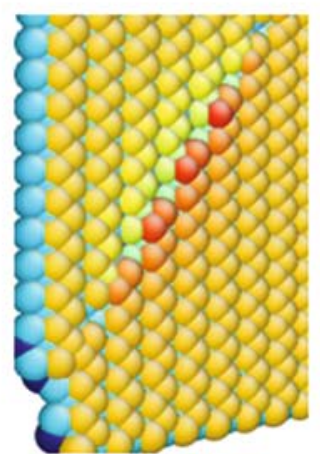

(b-3)

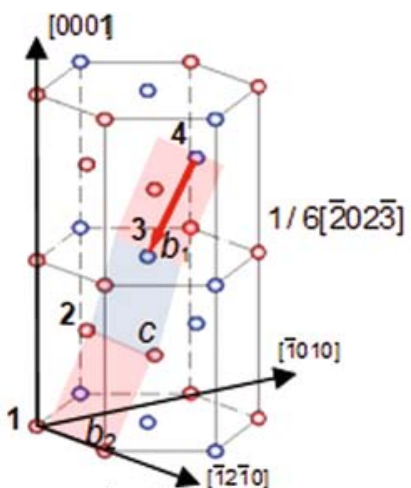

(c-1)

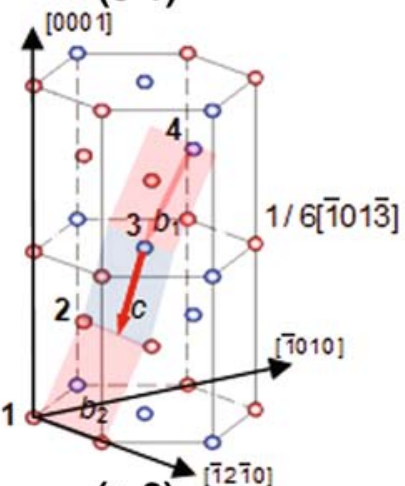

(c-2)

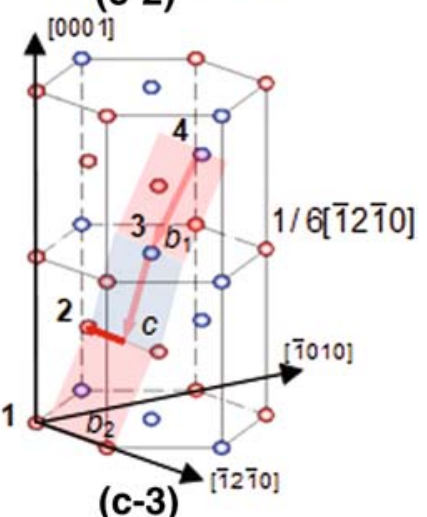

(c-3)

Fig. 5 Atomistic configuration of magnesium single crystal under $c$-axis compression at different time steps: a AtomEye visualization (identified by CNA); b AtomEye visualization (identified by coordinates); $\mathbf{c}$ slip vectors of the three steps

model, it is easy to calculate that the Schmid factor of the $(30 \overline{3} 2)$ plane with regard to the loading axis is 0.086 . While for the $(30 \overline{3} 4)$ plane, the Schmid factor is 0.47 . Therefore, we can assume that the $(30 \overline{3} 4)$ slip is easier to occur than the $(30 \overline{3} 2)$ slip due to the higher Schmid factor. As shown in Fig. 5(a-1), (b-1), when the value of strain is up to $8.9 \%$, it is found that the slip on $(30 \overline{3} 4)$ plane starts at the free surface, and a non-basal stacking fault follows. The slip vector of $1 / 6[\overline{2} 02 \overline{3}]$ is marked in Fig. 5(c-1). As shown in Fig. 5(a-2), (b-2), the second slip on (30) 2 ) plane occurs after a slight increase of $0.2 \%$ strain, and the slip vector of $1 / 6[\overline{1} 01 \overline{3}]$ is displayed in Fig. 5(c-2). And soon the last slip step of $1 / 6[\overline{1} 2 \overline{1} 0]$ dislocation, which is indicated in Fig. 5(c-3), follows to fulfill the full $1 / 3[\overline{2} 11 \overline{3}]$ slip. As shown in Fig. 5(a-3), we can see that the stacking fault disappears after the last slip occurs and the hcp structure is recovered. Meanwhile, a step with Burgers vector $1 / 6[\overline{1} 2 \overline{1} 0]$ is left at the surface along the $[\overline{1} 2 \overline{1} 0]$ direction. Thus, the slip path in our simulation is consistent with the path 3 described in Sect. 3.1. This result is also consistent with our previous MD simulation [19] that the pyramidal I $\langle c+a\rangle$ slip can be obtained by $1 / 3[11 \overline{2} \overline{3}] \rightarrow 1 / 6[20 \overline{2} \overline{3}]+$ $1 / 6[02 \overline{2} \overline{3}]$ in perfect magnesium single crystal under compression. However, due to the third step $1 / 6[\overline{1} 2 \overline{1} 0]$ slip 
followed very quickly, the decomposition of $1 / 6[\overline{2} 20 \overline{3}]$ into $1 / 6[\overline{1} 01 \overline{3}]$ and $1 / 6[\overline{1} 2 \overline{1} 0]$ was not observed in previous work with the higher strain rate.

\section{Conclusions}

In summary, through the calculations of GSFEs, the $\gamma$ surfaces in pyramidal I $\{10 \overline{1} 1\}$ and its adjacent planes $\{30 \overline{3} 4\}$ and $\{30 \overline{3} 2\}$ are computed using embedded-atom-method (EAM) method. The optimal slip paths can be predicted. The minimum energy path of $\langle 11 \overline{2} 3\rangle$ glide on $\{10 \overline{1} 1\}$ plane may occur in the ideal model by $1 / 6[\overline{1} 012]+1 / 12[1 \overline{4} 32]$ $+1 / 4[\overline{1012}]$. When the volume expansion and other factors are added to the simulation, the slip on $\{30 \overline{3} 4\}$ and $\{30 \overline{3} 2\}$ planes is more reasonable. Because of the difference of initial slip plane and direction, the probable paths can be approximately written as $1 / 6[\overline{2} 02 \overline{3}]+1 / 6[\overline{1} 01 \overline{3}]+1 / 6[\overline{1} 2 \overline{1} 0] \rightarrow$ $1 / 3[\overline{2} 11 \overline{3}]$ and $m / 6[\overline{2} 023]+((1-m) / 6[\overline{2} 023]+1 / 6[\overline{1} 2 \overline{1} 0])$ $+1 / 6[\overline{1} 013] \rightarrow 1 / 3[\overline{2} 113](m \approx 2 / 3)$. Moreover, the atomistic structure evolution of magnesium single crystal under $c$-axis compression at low temperature is simulated to test the GSFEs result. The atomic simulation evolution results by molecular dynamics method conform well to the theoretical analysis of GSFEs.

Acknowledgments This work was supported by the Fundamental Research Funds for the Central Universities of China (No. 2014YJS092) and the National Natural Science Foundation of China (No. 11372032).

\section{References}

[1] H. Yoshinaga, R. Horiuchi, Trans. JIM 4, 1 (1963)

[2] C.M. Byer, B. Li, B.Y. Cao, K.T. Ramesh, Scr. Mater. 62, 536 (2010)

[3] B. Li, P.F. Yan, M.L. Sui, E. Ma, Acta Mater. 58, 173 (2010)

[4] A. Galiyev, R. Kaibyshev, G. Gottstein, Acta Mater. 49, 1199 (2001)

[5] E. Lilleodden, Scr. Mater. 62, 532 (2010)

[6] S. Sandlöbes, M. Friák, J. Neugebauer, D. Raabe, Mater. Sci. Eng., A 576, 61 (2013)
[7] S. Ando, T. Gotoh, H. Tonda, Metall. Mater. Trans. A 33, 823 (2002)

[8] J.R. Morris, K.M. Ho, K.Y. Chen, G. Rengarajan, M.H. Yoo, Model. Simul. Mater. Sci. Eng. 8, 25 (2000)

[9] B. Syed, J. Geng, R.K. Mishra, K.S. Kumar, Scr. Mater. 67, 700 (2012)

[10] R.L. Bell, R.W. Cahn, Proc. R. Soc. Lond. A 239, 494 (1957)

[11] P.B. Price, J. Appl. Phys. 32, 1750 (1961)

[12] Y.F. Guo, S. Xu, X.Z. Tang, Y.S. Wang, S. Yip, J. Appl. Phys. 115, 224902 (2014)

[13] Y. Minonishi, S. Ishioka, M. Koiwa, S. Morozumi, M. Yamaguchi, Philos. Mag. A 43, 1017 (1981)

[14] D.J. Bacon, M.H. Liang, Philos. Mag. A 53, 163 (1986)

[15] M.H. Liang, D.J. Bacon, Philos. Mag. A 53, 181 (1986)

[16] M.H. Liang, D.J. Bacon, Philos. Mag. A 53, 205 (1986)

[17] H. Numakura, Y. Minonishi, Philos. Mag. A 62, 525 (1990)

[18] H. Numakura, Y. Minonishi, M. Koiwa, Philos. Mag. A 62, 545 (1990)

[19] B. Li, E. Ma, Philos. Mag. 89, 1223 (2009)

[20] Y.F. Guo, X.Z. Tang, Y.S. Wang, Z.D. Wang, S. Yip, Acta Metall. Sin. (Engl. Lett.) 26, 75 (2013)

[21] Y. Tang, J.A. El-Awady, Acta Mater. 71, 319 (2014)

[22] J.R. Morris, J. Scharff, K.M. Ho, D.E. Turner, Y.Y. Ye, M.H. Yoo, Philos. Mag. A 76, 1065 (1997)

[23] E.B. Tadmor, N. Bernstein, J. Mech. Phys. Solids 52, 2507 (2004)

[24] J.A. Yasi, T. Nogaret, D.R. Trinkle, Y. Qi, L.G. Hector Jr, W.A. Curtin, Modell. Simul. Mater. Sci. Eng. 17, 055012 (2009)

[25] T. Nogaret, W.A. Curtin, J.A. Yasi, L.G. Hector Jr, D.R. Trinkle, Acta Mater. 58, 4332 (2010)

[26] Z. Pei, L.F. Zhu, M. Friák, S. Sandlöbes, J.V. Pezold, H.W. Sheng, C.P. Race, S. Zaefferer, B. Svendsen, D. Raabe, J. Neugebauer, New J. Phys. 15, 043020 (2013)

[27] C. Wang, H.Y. Wang, H.Y. Zhang, X.L. Nan, E.S. Xue, Q.C. Jiang, J. Alloys Compd. 575, 423 (2013)

[28] J.F. Devlin, J. Phys. F: Met. Phys. 4, 1865 (1974)

[29] A. Couret, D. Caillard, Acta Metall. 33, 1455 (1985)

[30] D.-H. Kim, F. Ebrahimi, M.V. Manuel, J.S. Tulenko, S.R. Phillpot, Mater. Sci. Eng., A 528, 5411 (2011)

[31] I.P. Jones, W.B. Hutchinson, Acta Metall. 29, 951 (1981)

[32] S. Plimpton, J. Comp. Phys. 117, 1 (1995)

[33] X.Y. Liu, P.P. Ohotnicky, J.B. Adams, C.L. Rohrer, R.W. Hyland Jr, Surf. Sci. 373, 357 (1997)

[34] G. Kresse, J. Furthmüller, Comput. Mater. Sci. 6, 15 (1996)

[35] G. Kresse, J. Furthmüller, Phys. Rev. B 54, 11169 (1996)

[36] Y.F. Guo, Y.S. Wang, H.G. Qi, D. Steglich, Acta Metall. Sin. (Engl. Lett.) 23, 370 (2010)

[37] H.G. Qi, Y.F. Guo, X.Z. Tang, S. Xu, Acta Metall. Sin. (Engl. Lett.) 24, 487 (2011)

[38] D. Faken, H. Jónsson, Comp. Mater. Sci. 2, 279 (1994) 